\title{
A case study of a radially polarized Pc4 event observed by the Equator-S satellite
}

\author{
R. Cramm ${ }^{1}$, K. H. Glassmeier ${ }^{1}$, C. Othmer ${ }^{1}$, K. H. Fornacon ${ }^{1}$, H. U. Auster ${ }^{1}$, W. Baumjohann ${ }^{2}$, E. Georgescu ${ }^{2,3}$ \\ ${ }^{1}$ Institut für Geophysik und Meteorologie, Technische Universität, Mendelssohnstr. 3, D-38106 Braunschweig, Germany \\ ${ }^{2}$ Max-Planck-Institut für extraterrestrische Physik, PO Box 1603, D-85740 Garching, Germany \\ ${ }^{3}$ Institute of Space Sciences, Bucharest, Romania
}

Received: 8 February 1999 / Revised: 22 November 1999 / Accepted: 30 November 1999

\begin{abstract}
A $16 \mathrm{mHz}$ Pc4 pulsation was recorded on March 17, 1998, in the prenoon sector of the Earth's magnetosphere by the Equator-S satellite. The event is strongly localized in radial direction at approximately $L=5$ and exhibits properties of a field line resonance such as an ellipticity change as seen by applying the method of the analytical signal to the magnetic field data. The azimuthal wave number was estimated as $m \approx 150$. We discuss whether this event can be explained by the FLR mechanism and find out that the change in ellipticity is more a general feature of a localized Alfvén wave than indicative of a resonant process.
\end{abstract}

Key words: Magnetospheric physics (MHD waves and instabilities)

\section{Introduction}

Ultralow-frequency (ULF) pulsations are the manifestation of magnetohydrodynamic (MHD) plasma waves (Alfvén, 1942; Dungey, 1954). In the terrestrial magnetosphere, they have been observed by groundbased magnetometers (e.g. Glassmeier, 1980), by satellites (e.g. Cummings et al., 1969) and later by radar (e.g. Walker et al., 1979; Fenrich et al., 1995). Further studies of ULF waves have revealed more details of their properties and occurrence (e.g. Anderson et al., 1990; Takahashi and Anderson, 1992).

Characteristics of ULF pulsations such as frequency, wave number, localization, and polarization depend on the excitation mechanism. Generally speaking, ULF waves derive their energy from either internal magnetospheric sources or external sources, i.e. the solar wind. Plasma instabilities such as drift mirror (Hasegawa,

Correspondence to: C. Othmer

e-mail: c.othmer@tu-bs.de
1969; Woch et al., 1988) and bounce resonance instability (Southwood et al., 1969; Southwood and Kivelson, 1982) are discussed as an internal excitation mechanism for ULF waves. They allow a transformation of particle energy to wave energy. A prominent external excitation mechanism for ULF waves is the KelvinHelmholtz instability generating surface waves at the magnetopause (e.g. Fujita et al., 1996; Engebretson et al., 1998). Such waves propagate into the magnetosphere as isotropic fast modes and can couple resonantly to shear Alfvén waves via the field line resonance (FLR) process (Tamao 1965; Chen and Hasegawa, 1974; Southwood, 1974). This process has become a paradigm in understanding the features of a majority of observable ULF pulsations in the Pc3-5 range.

Polarization characteristics of ULF waves have been used as an indicator for possible excitation mechanisms. Toroidal pulsations are often explained in terms of the FLR theory (Anderson, 1993, and references therein) while radially polarized events were connected with magnetospheric plasma instabilities (Singer et al., 1982; Engebretson et al., 1992). The relation between radially polarized pulsations and FLR has recently been discussed (Ding et al., 1995; Fenrich et al., 1995) and deserves further attention.

To explore the magnetospheric dynamics in different regions, the Equator-S satellite was launched on December 2, 1997. It forms a part of the ISTP (International Solar-Terrestrial Physics) program and its final orbit resides close to the magnetospheric equator. The orbital and spin period is $22.3 \mathrm{~h}$ and $1.5 \mathrm{~s}$, respectively. With an apogee of more than $11 R_{E}$ and a perigee of only $500 \mathrm{~km}$ above the Earth, the orbit is highly eccentric and thus covers a wide range of $L$-shells from $L \approx 1.1$ to $L \approx 12$.

We present a Pc4 pulsation event whose characteristics are reminiscent to a radially polarized FLR event. Since neither electric field nor plasma measurements are available for the time interval of interest, we concentrate fully on the magnetic field data and show how the azimuthal wave number $m$ of the event can be estimated 
just by using Maxwell's equations. Finally, we discuss how the event fits into present theories on the excitation of ULF pulsations.

\section{The event}

The magnetic field data for this study were obtained from the Equator-S magnetometer experiment. This experiment consists of two units with a pair of threeaxes fluxgate magnetometers each. The sensors of the primary and the redundant units are mounted on two rigid booms, with the main sensor located at the end of the $1.8 \mathrm{~m}$ boom and the other $50 \mathrm{~cm}$ further inboard. They automatically switch between six ranges from 256 to $48600 \mathrm{nT}$ with a sampling rate up to $128 \mathrm{~Hz}$. We use spin averaged data with a time resolution of $1.5 \mathrm{~s}$ as we focus our interest on low-frequency processes.

On March 17, 1998, between 0935 UT and 0945 UT the Equator-S satellite recorded magnetic field oscillations with a period of $66 \mathrm{~s}$. At this time the spacecraft was on an inbound passage, $9^{\circ}$ off the magnetic equator, at a distance of about $4.7 R_{E}$ at $0830 \mathrm{LT}$. The spacecraft velocity in radial (azimuthal) direction was about 3.5 (2.5) $\mathrm{km} / \mathrm{s}$. The magnetic field perturbations are presented in a mean field field aligned (MFA) coordinate system in Fig. 1. The $z$-direction is defined as the direction of the background magnetic field, obtained by a 2 nd order polynomial fit to a half hour interval around each data point. Using the spacecraft's position vector $\vec{R}$, the $y$-direction of the MFA system is obtained by $\vec{y}=\vec{z} \times \vec{R}$ and points azimuthally eastward. The $x$-direction completes the orthogonal right-hand system, $\vec{x}=\vec{y} \times \vec{z}$, and points radially outward as long as the spacecraft is close to the magnetic equator. To emphasize the field perturbations, the background magnetic field $B_{0}$ was subtracted. Thus, only the fluctuating component $\vec{b}=\vec{B}-\overrightarrow{B_{0}}$ is used.

The Pc4 event shows up mainly in the radial component $b_{x}$ and exhibits only minor fluctuations in the $b_{z}$ component. Thus, the wave has a strong Alfvénic character with a dominating transverse polarization. The frequency of $15 \mathrm{mHz}$ is not typical for a fundamental eigenmode at $L \approx 5$. Results from ground measurements by Waters et al. (1991) suggest this frequency may be more typical of $L \approx 3$. Since the Equator-S spacecraft is located $9^{\circ}$ off the magnetic equator the wave could be a second harmonic. This assumption is consistent with studies by Singer et al. (1982) or Takahashi and Anderson (1992), where dayside Pc4 waves with similar frequency and polarization have been interpreted as second harmonic wave mode.

To achieve further information about the perturbation we use Gabor's (1946) method of the analytical signal (see also Glassmeier, 1980) that allows to compute the instantaneous amplitude, phase, and frequency. For a given monochromatic signal $x(t)$ the complex or analytic signal $z(t)$ is given by

$z(t)=x(t)-i \frac{1}{\pi} \int_{-\infty}^{+\infty} \frac{x(\tau)}{t-\tau} \mathrm{d} \tau$

where the imaginary part is known as the Hilbert transform of $x(t)$. For the instantaneous amplitude $A(t)$, phase $\phi(t)$ and angular frequency $\omega(t)$ the relations

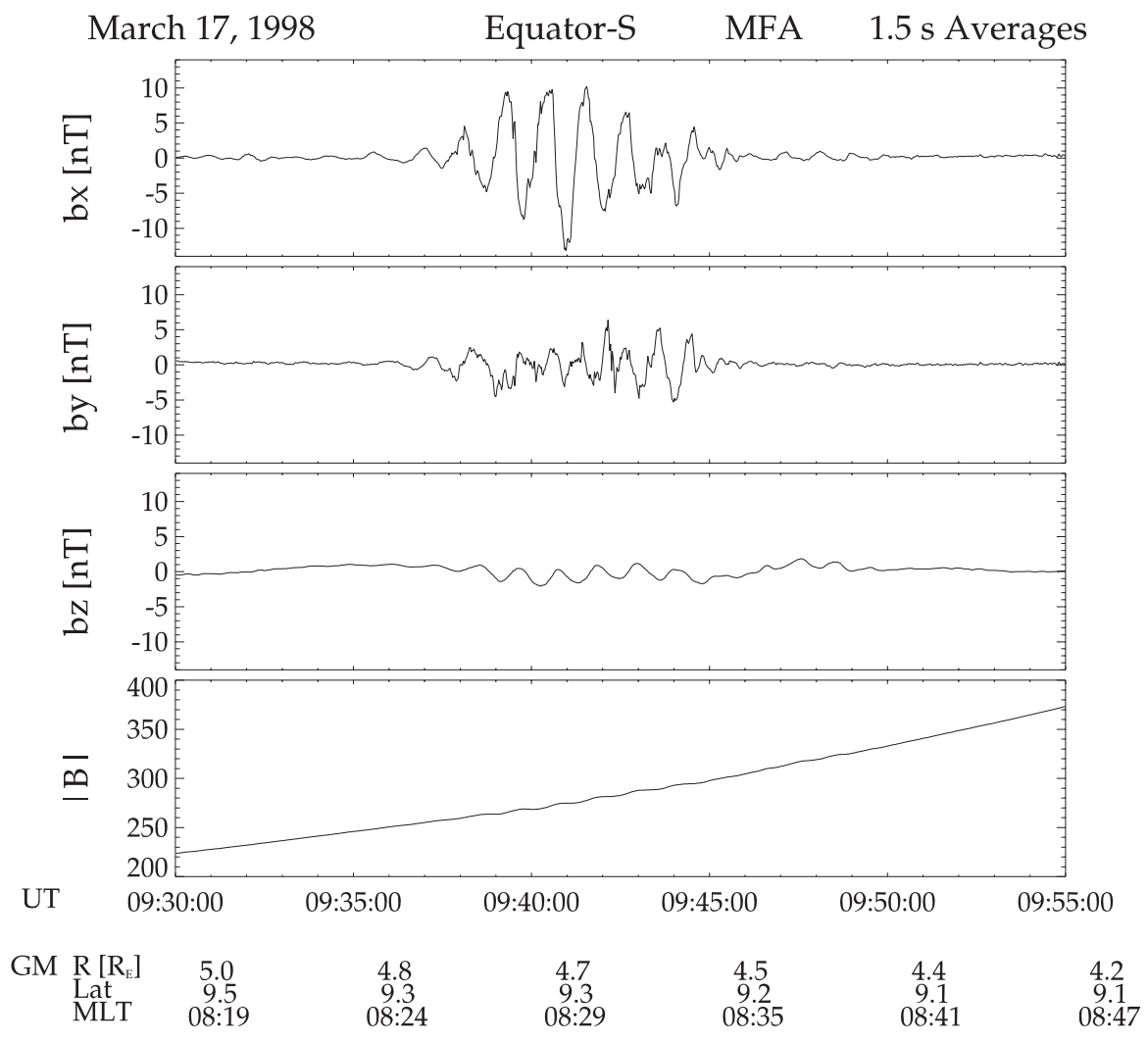

Fig. 1. The magnetic field perturbation presented in a mean field aligned coordinate system (see text for explanation). Note, that in the bottom graph the absolute value of the magnetic field is shown, while in the top three graphs the background magnetic field was subtracted. The position is given in geomagnetic coordinates 
$A(t)=\sqrt{x^{2}(t)+y^{2}(t)}$

$\phi(t)=\arctan (y(t) / x(t))$

$\omega(t)=\frac{\mathrm{d}}{\mathrm{d} t} \phi(t)$

hold, with $y(t)$ denoting the Hilbert transform of $x(t)$. With the amplitude and phase values of two components of the signal, it is also possible to compute the instantaneous ellipticity $\epsilon$ in the way given by Born and Wolf (1975).

Results of such an analytical-signal-analysis are shown in Fig. 2. It can be seen that the amplitude of the $b_{x}$-perturbation resembles a strongly localized distri-
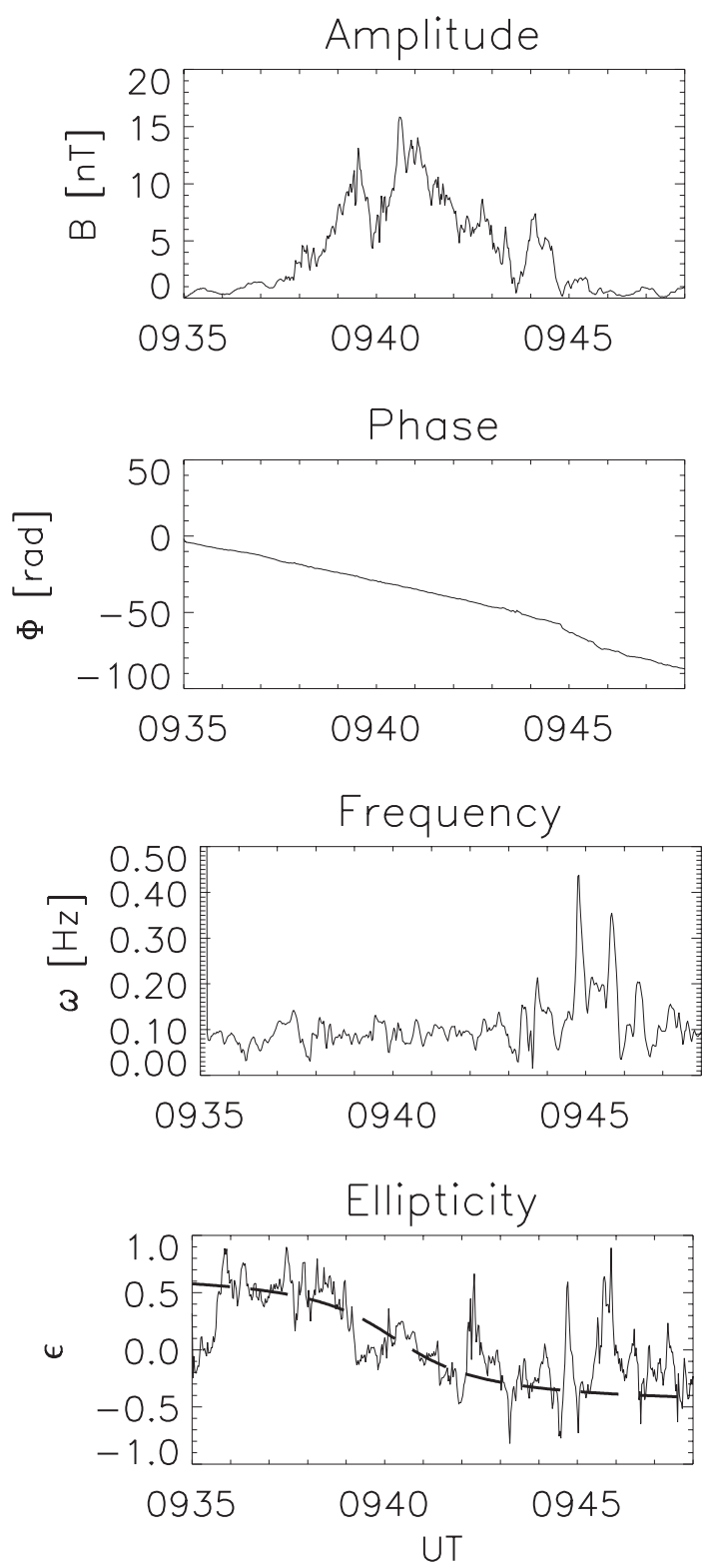

Fig. 2. The instantaneous amplitude and phase $\Phi$ of the $b_{x}$ perturbation computed with the analytical signal. Time differentiation of the phase yields the instantaneous frequency $\omega$. In the bottom graph an arctan-function (dashed line) approximates the ellipticity $\epsilon$ of the signal bution. Assuming that the amplitude distribution is due to a transit through a spatial structure, a half width of

$\sigma \approx 0.1 R_{E}$

can be determined. The frequency remains constant for the duration of the event, while the ellipticity $\epsilon$ resembles an arctan-function

$\epsilon \sim \arctan \left(\frac{x-x_{0}}{\sigma / 2}\right)$.

The signal turns from left-hand to right-hand polarization and is radially linearly polarized shortly before 0941 UT when $\epsilon=0$. At the same time the amplitude distribution shows its maximum.

A closer inspection of Fig. 2 indicates a fine structure of the signal with two maxima in the amplitude of the wave, a smaller one between 0939 and 0940 UT and the larger one between 0940 and 0941 UT. Looking at the ellipticity we also see that the value becomes negative for both time intervals. Such a double-resonance structure was observed previously by Nielsen and Allan (1983) and is attributed to the influence of non-uniform ionospheric conductivities and fine structures in the magnetospheric density (Glassmeier, 1984; McDiarmid, 1985). In the present study, however, we are not inferring this possibility any further.

\section{Discussion}

The results of Fig. 2 suggest that the event is a result of a resonant mode coupling process. The Alfvénic character, the radial localization and the change of ellipticity are typical features of a FLR as it was described theoretically by Southwood (1974). To study this hypothesis further we estimate the azimuthal wave number, using only the data of the spacecraft's observation. As the wave is mainly transverse polarized, we assume $b_{z}=0$ for the time being. Thus, from $\nabla \cdot \vec{b}=0$ one gets

$\frac{\partial}{\partial x} b_{x}=-\frac{\partial}{\partial y} b_{y}$.

Assuming furthermore a plane wave approximation,

$\vec{b}=\left(b_{x}^{0}(x), b_{y}^{0}(x), 0\right) \exp \left\{i\left(k_{y} y+k_{z} z-\omega t\right)\right\}$,

we get from Eq. (5) an expression for the polarization of the transverse magnetic field perturbation:

$\frac{b_{y}^{0}}{b_{x}^{0}}=\frac{i}{k_{y} b_{x}} \frac{\partial b_{x}^{0}}{\partial x}$.

The azimuthal wave number $m$ is related to $k_{y}$ by $m=k_{y} r$, where $r$ denotes the distance of the wave from the Earth's centre. Approximating

$\frac{\partial b_{x}^{0}}{\partial x} \approx \frac{b_{x}^{0}}{\sigma}$

with $\sigma$ as the width of localization, we get from Eq. (7)

$|m|=\frac{r}{\sigma}\left|\frac{b_{x}^{0}}{b_{y}^{0}}\right|$. 
This expression allows an estimation of the azimuthal wave number and is applicable to the presented event. From Fig. (1) the amplitude ratio $b_{x}^{0} / b_{y}^{0}$ is determined to $\approx 3$. Using Eq. (3) and considering a distance of the observation of $4.7 R_{E}$, Eq. (9) yields a value for $m$ of about 150 , suggesting a high $m$ FLR. It is noteworthy that a large $m$ value as derived from Eq. (9) is due to the small value of the amplitude distribution $\sigma$. Thus, large $m$ and radial polarization only go together with a strong localization of the wave field in the radial direction.

With the movement of the spacecraft being not only in radial but also in azimuthal direction, the question arises how much the azimuthal harmonic structure of the original signal was altered by the Doppler effect. The Doppler related frequency shift $\Delta \omega$ can be calculated as

$\Delta \omega=k_{y} v_{y}^{s / c}=\frac{m}{r} v_{y}^{s / c}$,

where $v_{v}^{s / c}$ denotes the azimuthal component of the spacecraft velocity. With $m$ as determined already and the satellite velocity of $2.5 \mathrm{~km} / \mathrm{s}$, a frequency shift of $\Delta \omega=12 \mathrm{mHz}$ results. As compared to the $95 \mathrm{mHz}$ of the recorded signal, we thus conclude that the azimuthal movement of the satellite has not altered the original structure of the pulsation significantly.

With the interpretation of the event as a high $m$ FLR we are faced with a problem when considering the coupling strengths in FLR regions. As discussed by Speziale and Catto (1977) and Kivelson and Southwood (1986), the coupling efficiency between the fast mode and the Alfvén wave can be described by a dimensionless coefficient $q$. These authors find that the coupling efficiency maximizes for a specific value of $q$, depending on characteristics such as the wave vector and also on plasma conditions like the Alfvén velocity and its gradient. Using a dipole magnetosphere model Lee and Lysak (1990) have determined this value for typical conditions in the Earth's magnetosphere. They find that $q$ maximizes for $m \approx 3$, and very little coupling occurs for much smaller or larger values of $m$, confirming a previous study by Poulter et al. (1988). This is certainly a contradiction to the interpretation that the high $m$ Alfvén wave is the result of resonant coupling between the fast and Alfvén mode and the question concerns which excitation process the event can be connected to.

Recent ideas of Fenrich et al. (1995) and Fenrich and Samson (1997) give reason to speculate that despite of the poor coupling efficiency the FLR process may well play an important role for the high $m$ case. They suggest that there is only weak coupling between the external driven fast mode and the Alfvén wave, but secondary amplification processes such as internal plasma instabilities make a considerable growth of the Alfvén wave possible. These ideas overcome the problem of the poor coupling efficiency of high $m$ waves so that the presented event remains a candidate for a FLR. A verification of a possible secondary amplification processes make further studies involving plasma data necessary.

However, it may be questioned whether the observed event is due to a field line resonance at all. We considered the change of polarization as an indicator for such a resonant coupling process. The question arises whether this conclusion is cogent. Indeed, the change of polarization can be explained for localized Alfvén waves by a more general approach. A localized amplitude such as in the top graph of Fig. 2 can be approximated by a Gaussian. Having this in mind, it is clear that the change of polarization must occur because the spatial derivative of the Gaussian and thus of $b_{x}^{0}$ in Eq. (7) changes sign over the centre of the localized event. This behaviour can be found in Fig. 2, where the change of polarization occurs at the same position where the amplitude distribution reaches its maximum, i.e. when its spatial derivative changes sign.

Note that the determination of the azimuthal wave number in Eq. (9) and the resulting effect of a change of polarization of the wave were derived by fundamental equations, only assuming a location of the wave event and not assuming any specific resonant process causing the localization. Similar arguments apply for ground based observations of localized Alfvén waves, where the influence of the ionosphere has to be taken into account (Glassmeier, 1992). Thus, the spatial change of polarization is an inherent characteristic of any localized Alfvén wave, determined by Maxwell's equations. By itself, it cannot be considered as a sufficient criteria for FLR.

Possible alternative mechanisms are ring current instabilities according to earlier discussions by other authors for radially polarized pulsations (Singer et al., 1982; Engebretson et al., 1992). For an unambigiuous identification of FLRs a more detailed examination of the possible excitation mechanism is necessary. On the one hand plasma data would allow investigations about the dynamics and the distribution function of the plasma to verify a possible excitation of a pulsation via a plasma instability. Electric field data together with magnetic field data on the other hand allow the computation of Poynting fluxes to identify FLRs by the energy flow into the resonance region.

\section{Summary and conclusions}

For the Pc4 wave event presented we could estimate from the localization and the ratio of the transverse perturbations an azimuthal wave number of $m \approx 150$. Together with the other characteristics, this event shows all the properties which we would expect of a high $m$ FLR, i.e. the sharp radial localization, the Alfvénic character with a predominantly radial polarization and the change of ellipticity over the centre of resonance. However, resonant coupling efficiencies are expected to be small for large $m$-values. This requires caution in interpretating this event as a FLR. We would like to emphasize, however, that a change of phase is a necessary condition for a FLR but not a sufficient one. We have shown that Maxwell's equations do not allow any other behaviour for a localized Alfvén wave than a reversal of ellipticity. That means that the character of a localized Alfvén wave obtained by magnetic field data alone does not allow us to draw unambiguous conclu- 
sions on the excitation of the wave. For this purpose additional information on plasma and electric field data are necessary.

Acknowledgements. This work was financially supported by the Deutsche Forschungsgemeinschaft.

The Editor-in-chief thanks M. Lester and B. Fraser for their help in evaluating this paper.

\section{References}

Alfvén, H., On the existence of electromagnetic - hydromagnetic waves, Ark. Mat. Astron. Fys., 29B(2), 1942.

Anderson, B. J., Statistical studies of Pc 3-5 pulsations and their relevance for possible source mechanisms of ULF waves, Ann. Geophysicae, 11, 128, 1993.

Anderson, B. J., M. J. Engebretson, S. P. Prounds, L. J. Zanetti, and T. A. Potemra, A statistical study of Pc3-5 pulsations observed by the AMPTE/CCE magnetic field experiment 1 . Occurrence distribution, J. Geophys. Res., 95, 10 495, 1990.

Born, M., and E. Wolf, Principles of optics, 5th edn., Pergamon, Oxford, 1975.

Chen, L., and A. Hasegawa, A theory of long-periodic magnetic pulsations, 1, Steady state excitation of field line resonance, J. Geophys. Res., 79, 1024, 1974.

Cummings, W. D., R. J. O'Sullivan, and P. J. Jr Coleman, Standing Alfvén waves in the magnetosphere, J. Geophys. Res., 74, 778, 1969.

Ding, D. Q., R. E. Denton, M. K. Hudson, and R. L. Lysak, An MHD simulation study of the poloidal mode field line resonance in the Earth's magnetosphere, J. Geophys. Res., 100, 63, 1995.

Dungey, J. M., Electrodynamics of the outer magnetosphere, Sci. Rep. 69, Pa. State Ionos. Res. Lab., University Park, 1954.

Engebretson, M. J., D. L. Murr, K. N. Erickson, R. J. Strangeway, D. M. Klumpar, S. A. Fuselier, L. J. Zanetti, and T. A. Potemra, The spatial extent of radial magnetic pulsation events observed in the dayside near geosynchronous orbit, J. Geophys. Res., 97, $13741,1992$.

Engebretson, M. J., K. H. Glassmeier, M. Stellmacher, W. J. Hughes, and H. Lühr, The dependence of high-latitude Pc5 wave power on solar wind velocity and on the phase of highspeed solar wind streams, J. Geophys. Res., 103, $26271,1998$.

Fenrich, F. R., and J. C. Samson, Growth and decay of field line resonances, J. Geophys. Res., 102, 20 031, 1997.

Fenrich, F. R., J. C. Samson, G. Sofko, and R. A. Greenwald, ULF high- and low-m field line resonances observed with the Super Dual Auroral Radar Network, J. Geophys. Res., 100, 21 535, 1995.

Fujita, S., K. H. Glassmeier, and K. Kamide, MHD waves generated by the Kelvin-Helmholtz instability in a nonuniform magnetosphere, J. Geophys. Res., 101, $27317,1996$.
Gabor, D., Theory of communication, J. Inst. Elec. Eng., 93, 429, 1946.

Glassmeier, K. H., Magnetometer array observations of a giant pulsation event, J. Geophys., 48, 127, 1980.

Glassmeier, K. H., On the influence of ionospheres with nonuniform conductivity distribution on hydromagnetic waves, J. Geophys. Res., 54, 125-137, 1984.

Glassmeier, K. H., Traveling magnetospheric convection twin-vortices: observation and theory, Ann. Geophysicae, 10, 547, 1992.

Hasegawa, A., Drift mirror instability in the magnetosphere, Phys. Fluids, 12, 2642, 1969.

Kivelson, M. G., and D. J. Southwood, Coupling of global magnetospheric MHD eigenmodes to field line resonances, J. Geophys. Res., 91, 4345, 1986.

Lee, D. H., and L. Lysak, Effects of azimuthal asymmetry on ULF waves in the dipole magnetosphere, Geophys. Res. Lett., 17, 53, 1990.

McDiarmid, D. R., On the possibility that nonuniform ionospheric conductivity can cause a pulsation double resonance, J. Geophys. Res., 90, 4398-4402, 1985.

Nielsen, E., and W. Allan, J. Geophys. Res., 88, 5760-5764, 1983.

Poulter, E. M., W. Allan, and G. J. Bailey, ULF pulsation eigenperiods within the plasmasphere, Planet. Space Sci., 36, 185, 1988.

Singer, H. J., W. J. Hughes, and C. T. Russell, Standing hydromagnetic waves observed by ISEE 1 and 2: radial extent and harmonic, J. Geophys. Res., 87, 3519, 1982.

Southwood, D. J., Some features of field line resonances in the magnetosphere, Planet. Space Sci., 22, 483, 1974.

Southwood, D. J., and M. G. Kivelson, Charged particle behaviour in low-frequency geomagnetic pulsations. 2. Graphical approach, J. Geophys. Res., 87, 1707-1710, 1982.

Southwood, D. J., J. W. Dungey, and R. J. Etherington, Bounce resonant interaction between pulsations and trapped particles, Planet. Space Sci., 17, 349-361, 1969.

Speziale, T., and D. J. Catto, Linear wave conversion in an unmagnetized collisionless plasma, Phys. Fluids, 20, 990, 1977.

Takahashi, K., and B. J. Anderson, Distribution of ULF Energy $(f<80 \mathrm{mHz})$ in the inner magnetosphere: a statistical analysis of AMPTE/CCE magnetic field data, J. Geophys. Res., 97, $10751,1992$.

Tamao, T., Transmission and coupling resonance of hydromagnetic disturbances in the non-uniform Earth's magnetosphere, Sci. Rep. Tohoku Univ., Ser. 5, 17(2), 43, 1965.

Walker, A. D. M., R. A. Greenwald, W. F. Stuart, and C. A. Green, STARE auroral radar observation of Pc5 geomagnetic pulsations, J. Geophys. Res., 84, 3373, 1979.

Waters, C. L., F. W. Menk, and B. J. Fraser, The resonance structure of low latitude Pc3 geomagnetic pulsations, Geophys. Res. Lett., 18, 2293, 1991.

Woch, J., G. Kremser, A. Korth, O. A. Pokhotelov, V. A. Pilipenko, Y. M. Nezlina, and E. Amaty, Curvature-driven drift mirror instability in the magnetosphere, Planet. Space Sci., 36, 383393, 1988. 